\title{
WristQue: A Personal Sensor Wristband
}

\author{
Brian D. Mayton, Nan Zhao, Matt Aldrich, Nicholas Gillian, and Joseph A. Paradiso \\ Responsive Environments Group, Massachusetts Institute of Technology Media Lab \\ 75 Amherst St., Cambridge, MA 02139 \\ \{bmayton,nzhao,maldrich,ngillian,joep\}@media.mit.edu
}

\begin{abstract}
WristQue combines environmental and inertial sensing with precise indoor localization into a wristband wearable device that serves as the user's personal control interface to networked infrastructure. WristQue enables users to take control of devices around them by pointing to select and gesturing to control. At the same time, it uniquely identifies and locates users to deliver personalized automatic control of the user's environment. In this paper, the hardware and software components of the WristQue system are introduced, and a number of applications for lighting and HVAC control are presented, using pointing and gesturing as a new human interface to these networked systems.
\end{abstract}

\section{INTRODUCTION}

Network control is increasingly common in the devices that people interact with in their daily lives. Modern commercial buildings commonly have computerized lighting, heating, cooling, entertainment, and other infrastructure systems. Recently, networked and computerized control has been expanding into people's homes as well. However, this network control is often underutilized due to poor user interfaces and limited sensor input. In fact, current building infrastructure systems can be more difficult for users to operate, as simple concepts like light switches have been replaced by complex control panels. Sensors, such as thermostats, have also remained on the walls, distant from where people are actually occupying the space.

WristQue is a personal wearable sensing and control device in the form of a wristband. It aims to increase the usefulness of infrastructure systems in two ways. First, by moving sensors off of the walls and onto people, automatic control can react to the conditions where the people actually are, rather than arbitrary locations on the wall. Similarly, moving controls to the user's wrist places them where they are always within reach, rather than forcing the user to find a control panel.

Second, WristQue uniquely identifies and locates users within the building. This provides a critical part of the context needed by automatic systems to make optimal control decisions. Each user can be associated with his or her personal preferences (perhaps automatically learned by the system) so that the building systems can adjust conditions to suit the users who are currently using various spaces within the building. Location also provides the critical context for more intuitive control of devices in the environment, making it easier for users to select controllable devices by proximity rather than by arbitrary zone numbers and identifiers.

Smartphones both provide a user interface for display and control, and include many of the same sensors as the WristQue. However, smartphones are generally kept in a pocket or bag, which limits their ability to sense the user's environment when they are not actively being operated. WristQue brings the sensors out to the wrist, where they may sometimes be momentarily obscured by clothing, but are not completely enclosed like a phone in a pocket. This allows WristQue to measure the conditions around the user more often than a phone would be able.

Performing control tasks on a smartphone requires retriev-

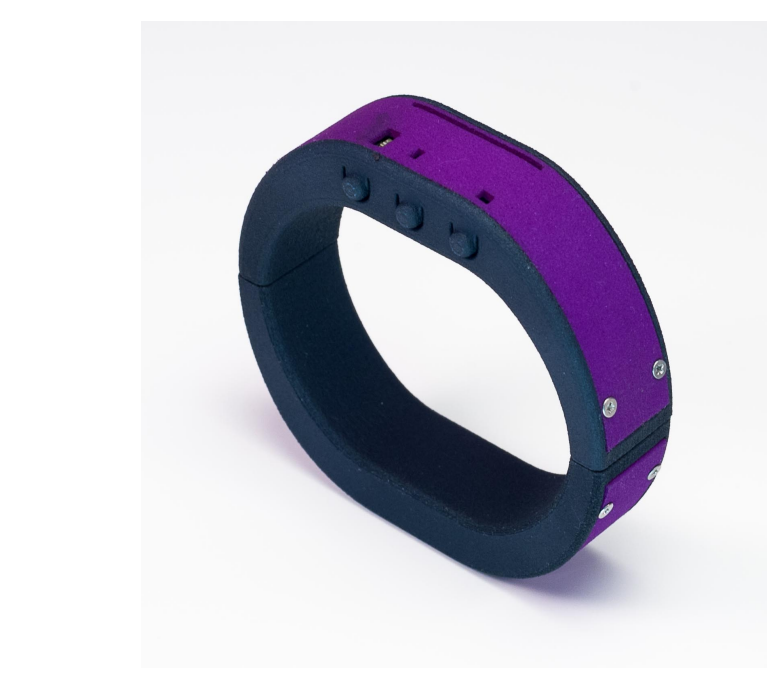

Fig. 1. The WristQue prototype device.

ing the device, waking and unlocking it, and finally launching an app specific to the task. While this is worthwhile for complex tasks where the affordances of a high-resolution display and touchscreen are desired, it's a lot of work just to turn on the lights, for example. The idea of WristQue as a user interface is to put controls for common, simple tasks where they are always within easy reach. WristQue's positioning also allows it to exploit intuitive arm gestures to form an easy-touse human interface.

WristQue explores multiple input modalities for control. Buttons form a very simple kind of user interface that users are already accustomed to on watches. With location sensing, the buttons can be made context-sensitive, such as a lightswitch that changes what it controls based on the user's proximity. However, overloading buttons with too many functions, or having too many buttons, can quickly make a user interface confusing.

To allow the user to select and control many devices in the environment, WristQue implements a pointing-based interface. Measuring the user's location and the orientation of his or her wrist enables WristQue to identify which controllable object in the environment the user is pointing toward. As other researchers have noted, pointing is a natural gesture for people to identify objects at a distance [1]. Once the user has selected an object to control, WristQue can use its inertial sensors to identify gestures as commands.

\section{RELATED WORK}

The wrist has long been a natural place for wearable technology. People have worn watches on their wrists since it became possible for mechanized clocks to be made sufficiently small. Watches have declined in popularity with the advent of cell phones, but are making a comeback as wearable information displays, some of which can be paired with cell 
phones, such as the Sony Smartwatch and the Pebble. Wristworn computers have also appeared as research and consumer products. The IBM Linux Watch [2] brought simple computing to the wrist. Microsoft's SPOT watch [3] was an early attempt at a wrist-worn device that augmented time with other data feeds using an FM radio network. Maurer's eWatch [4] is a wrist-worn research sensor platform; the authors briefly introduce the idea of a wearable device as a universal interface to smart environments, but mainly focus on activity recognition and classification. Notably, they cite the need for precise indoor localization. The TI EZ430-Chronos device [5] is another watch-like device with a suite of sensors, available as a development kit.

Other systems have used wearable sensors for monitoring the wearer and his or her environment. TRUSS [6] is a badgestyle sensor for measuring the worker's altitude and movement and monitoring environmental parameters to identify potentially hazardous conditions on construction sites. Many other researchers have also built wearable platforms for activity recognition and context-aware monitoring for a variety of applications [7]-[9].

WristQue's location-awareness builds on the idea of proxemic interfaces [10]. Pointing (using motion capture systems) was evaluated as an interface to large 2-dimensional displays in [1].

The WristQue hardware and system were first described in [11].

\section{SYSTEM DESCRIPTION}

The WristQue system consists of two parts: the WristQue wristband and the supporting infrastructure.

The wristband contains the sensors, radio, microprocessor, and battery. The electronics are split across three PCBs, both for expandability and to make the best use of the space inside the band. The mainboard contains the main CPU (ATxmega32A4), the buttons, and a secondary microcontroller (ATtiny40) that controls LEDs and has a capacitive touch peripheral. Another PCB contains the radio and antenna, and the final PCB, designed to be easily replaceable, contains the full sensor set.

The supporting infrastructure is comprised by the wireless network base stations, the server software, the systems that WristQue controls (such as the lighting system) and the indoor localization system.

\section{A. Wristband Design}

While many wearable sensor platforms have been designed, they are rarely in a form that people would be willing to wear outside of the context of a research study. Many wearable sensor nodes take the form of badges, which may work in corporate environments where employees are already required to wear badges. However, in a more casual environment like the Media Lab or a home, nobody wants to wear a badge on a regular basis. The wristband is an attempt to put a wearable sensor node into a form that people might be willing to wear regularly, similar to how people wear watches or jewelry on their wrists. We elected to use a neutral wristband design rather than trying to design a new watch, as people are often attached to their particular watch and are hesitant to replace it. The wristband allows the sensing to be added alongside an existing watch.

The prototype WristQue is designed to separate into two halves (fig. 2), one containing the sensors and electronics,

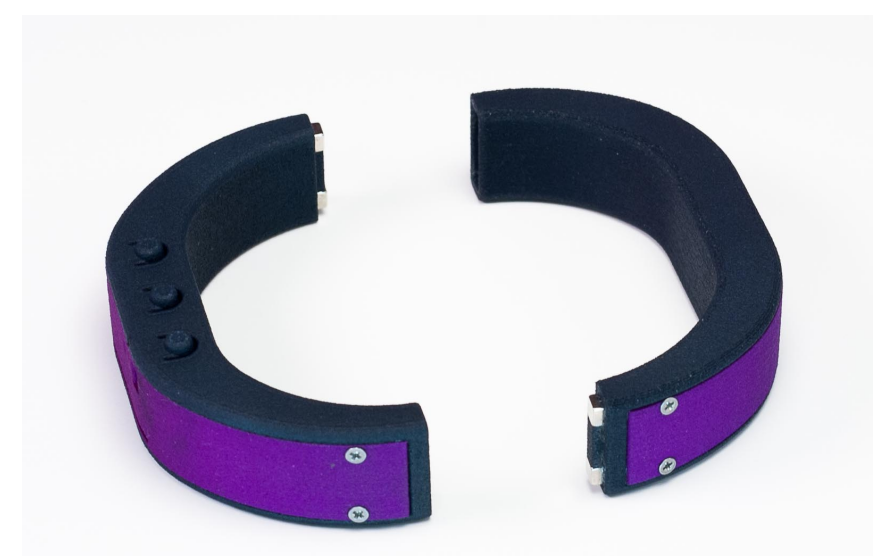

Fig. 2. The WristQue separated into its two halves. The electronics are in the top half (left), and the battery in the bottom (right).

and the other containing the battery. The two halves are held together by magnets, which also serve as the electrical contacts for connecting the battery to the electronics. The separability of the two halves allows the device to be easily placed around the wrist, and allows the battery to be removed and placed on a base for recharging.

The enclosure is made from 3D-printed plastic and is shaped and sized to balance a reasonably small and unobtrusive form with easy assembly and serviceability of the electronics inside. The ideal wristband device would be smaller and more flexible; however, further miniaturization and flexibility introduce design challenges that, while not complex, are not the focus of this work in its current state.

The 3D CAD model of the WristQue enclosure is parametrically driven by a small number of wrist measurements. This allows a custom enclosure to be printed for each person's wrist, providing a comfortable, but snug fit so that the WristQue cannot freely rotate on the wrist, which would add uncertainty to the measured wrist orientation.

\section{B. Network and Backend}

The WristQue wristbands communicate with a server computer through an 802.15.4 wireless network implemented on Atmel's AT86RF231 radio. The base stations are custom boards with USB connectors. When connected to a computer, they communicate over USB to the WristQue server software. They can also be plugged in to a power source only (such as a USB cell phone charger) to act as repeaters that forward packets back to the server. The base stations also collect signal strength information for beacon packets and send the data back to the server, which could be used as a coarse localization estimate in absence of the UWB system.

The WristQue server software is a collection of programs implemented in the Willow Garage ROS [12] pub/sub framework. This allows for rapid prototyping of new applications, as all of the data is readily accessible by any program in the system. Data from the sensors are also stored in a MySQL database where they can be accessed by other programs.

The server is also responsible for bridging between the WristQue and the systems it controls, such as the Lutron lighting system installed in our lab. 


\section{Indoor Localization}

WristQue is inspired by the idea that precise indoor localization will soon be readily available in commodity radio parts. Projects such as the Qualcomm Peanut [13] and Nokia HAIP [14] use UWB TDoA (time difference of arrival) and low-power Bluetooth AoA (angle of arrival), respectively. Both use low-power radios that can also be used for communication. No easily integrable parts are readily available at this time, however it is clear that such technology will be common in the coming years.

For the prototype WristQue, indoor localization is performed by a separate Ubisense [15] UWB real-time localization system. This requires the UbiSense antennas to be installed in the space, and for an additional tag to be affixed to the WristQue.

\section{Inertial/Orientation Sensing}

The WristQue senses its motion and orientation through four 3-axis sensors. High-resolution, high-bandwidth acceleration and angular rates are sensed by an Invensense MPU-6150 part. A Honeywell HMC5883L magentometer measures the magnetic field as a heading reference. These sensors are used when the user is actively interacting with the WristQue. They are configured to operate at $20 \mathrm{~Hz}$, feeding real-time inertial and magnetic measurements back to the server via the wireless network. The server uses the orientation filter described in [16] to estimate the orientation of the WristQue.

The sensor board also includes an Analog Devices ADXL346, which is a low-power accelerometer, efficient enough to remain continuously powered for low-bandwidth acceleration sensing. This part is configured with a sample rate of $12 \mathrm{~Hz}$ and has an internal FIFO, so it can accumulate a buffer of samples before waking the main CPU. The lowpower accelerometer is used to estimate the user's amount of physical activity, and to detect when the user might be trying to initiate a gesture (which indicates that it should switch on the higher-power inertial sensors).

The most significant challenge of indoor orientation sensing is determining the device's heading. The earth's magnetic field as sensed by a 3-axis magnetometer is used as a reference to keep the orientation estimate from drifting, but the magnetic field cannot be assumed to point north inside a building. Metal construction materials and equipment significantly distort the magnetic field. Metal objects, such as furniture, also distort the magnetic field, but at much shorter ranges [17]. Fig. 5 shows a 2-dimensional slice of the magnetic field in the lab space where the WristQue was tested. There are significant variations in the direction and magnitude of the field across a relatively small space.

\section{E. Environmental Sensing}

In addition to sensing the wearer's movements to create a control interface, WristQue also monitors the wearer's environment. The sensors on the prototype sensor board are chosen for applications in lighting and HVAC control.

Two light sensors measure the lighting conditions at the user's wrist. One is a color light sensor (Avago) but operates at a low sample rate. The other is a monochrome visible light sensor (ISL29003) with much higher bandwidth, which enables the WristQue to demodulate information encoded by flashing solid-state light sources fast enough to be invisible (see section IV-C).
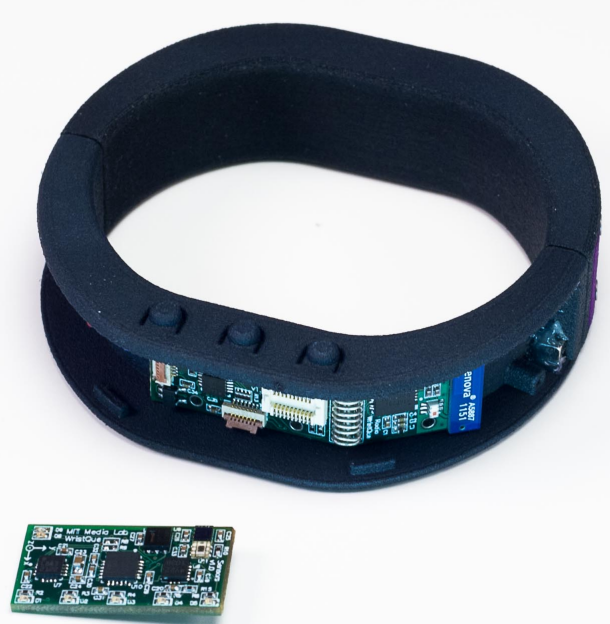

Fig. 3. The WristQue, open, with the sensor board removed. The sensor board plugs in to the rectangular connector on the mainboard in the band.

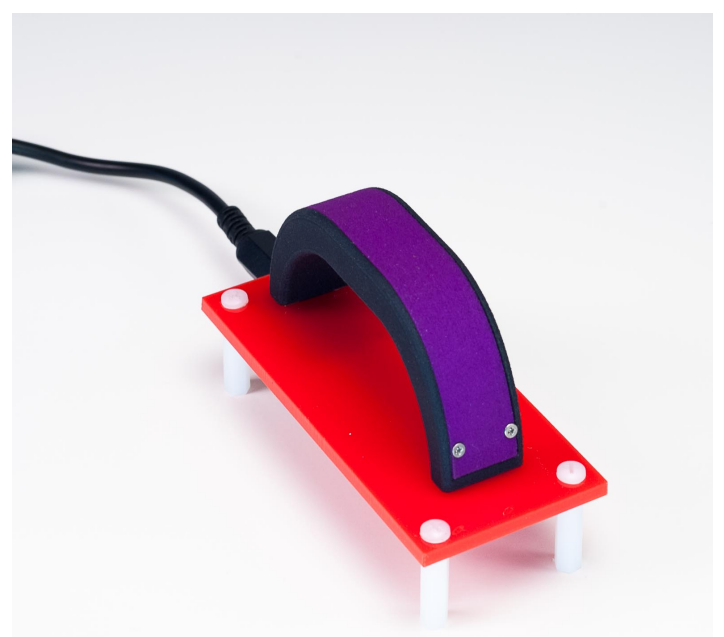

Fig. 4. The WristQue battery on its charging base. The $110 \mathrm{mAh}$ battery charges in an hour and lasts for over a week of normal use.

Temperature and humidity are sensed by a Sensirion SHT25 sensor.

\section{F. Power}

The WristQue is powered from a $110 \mathrm{mAh}$ battery in the bottom half of the band.

To manage power consumption, the WristQue defines three modes of operation, each using more power than the previous while providing additional functionality. The WristQue moves between these different modes as necessary for the application(s) currently running.

Sleep mode is automatically entered when the WristQue loses contact with its network. In this mode, the sensors are completely powered down. The CPU and radio periodically wake up and attempt to reassociate with the network. If reassociation is successful, then the WristQue transitions to sensing 
mode. The time between reassociation attempts is dynamically scaled such that the WristQue tries to reassociate frequently immediately after losing the network connection, but scales back to attempting every few minutes if multiple attempts fail. In sleep mode, the measured average current draw is approximately $131 \mu \mathrm{A}$. The quiescent current draw is roughly $120 \mu \mathrm{A}$, with an additional $11 \mu \mathrm{A}$ (average) consumed by radio communication for reassociation attempts. The WristQue can operate in sleep mode for approximately 35 days on a single charge.

In quiescent sensing mode, the environmental sensors are powered on every ten seconds and sampled. The resulting data are transmitted in a single packet via the wireless interface. In sensing mode, the low-power accelerometer is also enabled at a sample rate of $12.5 \mathrm{~Hz}$, with the CPU waking up every second to read the accelerometer's FIFO. The integrated and average acceleration are transmitted and reset every ten seconds with the rest of the sensor data. This mode provides the sensor data necessary for applications such as personalized automatic HVAC and comfort control. In sensing mode, the measured current draw averages $260 \mu \mathrm{A}$. The WristQue can operate in quiescent sensing mode for over two weeks on a single charge.

In interaction mode, the full IMU, including the 16bit accelerometer, the gyroscopes, and the magnetometer, is powered on and sampled at $20 \mathrm{~Hz}$. Packets are transmitted back via the wireless network at $10 \mathrm{~Hz}$. This mode consumes the most power $(14.3 \mathrm{~mA})$ so it is only entered when the user is actively pointing or gesturing. The WristQue can operate continuously for over 7 hours in interaction mode.

\section{APPLICATIONS}

\section{A. Personal Lightswitch}

The personal lightswitch application is a simple demonstration of how precise localization and readily accessible controls can provide an easy-to-use interface to building infrastructure. The application uses two buttons on the WristQue to adjust the lighting zone nearest to the user. One button raises the lights, while another button dims. Even this very simple application removes the need for the user to find the lighting control panel on the wall, know which zone number to control, and figure out which one of many buttons actually adjusts that zone.

\section{B. Pointing interface}

To enable the user to select and control a multitude of controllable devices in the environment, we developed an interface based on pointing and gesturing. Pointing is a natural gesture that people use to indicate specific objects around them.

The position and orientation of the WristQue device are used together to define a ray that extends along the user's arm. As the user points, the system computes the intersection of the ray with the planes of the walls and ceiling of the room. For the lighting control experiment, only the ceiling plane is used, with rectangular regions demarcating lighting zones that may be controlled. More generally, this approach could be extended to consider intersections with arbitrary 3D bodies representing controllable objects in the environment.

We conducted two tests with the pointing system in our lab space. The "room," for the purposes of these experiments, was a 6 by 14 meter region of a large lab space (fig. 5) with the ceiling 2.6 meters above the floor. The space includes work tables, desks, and shelving.

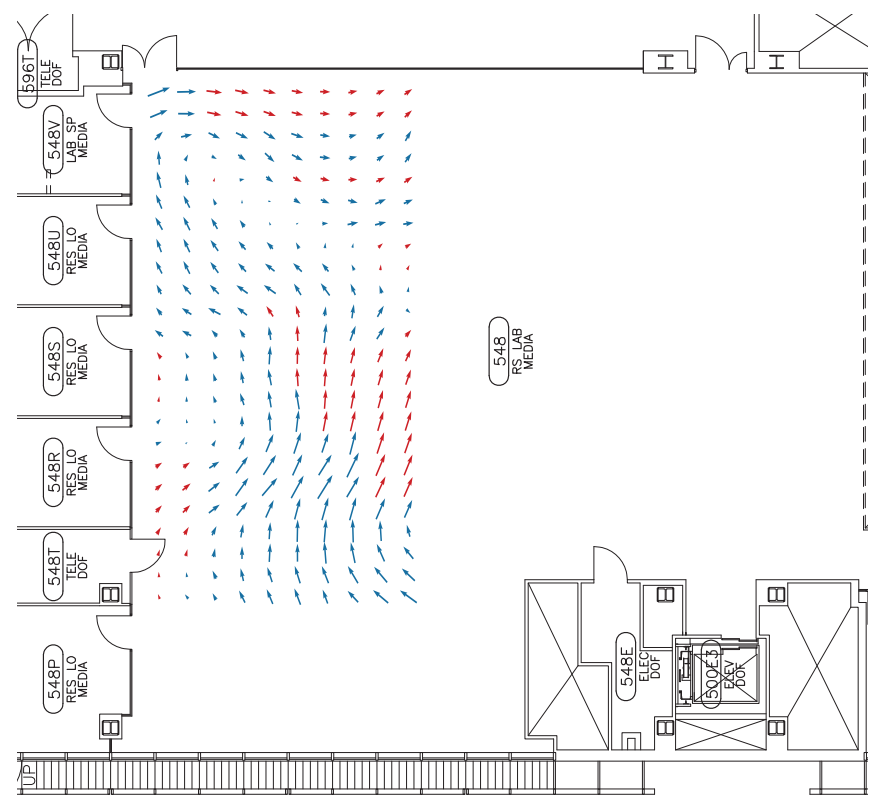

Fig. 5. Map of the space where the experiments were conducted, showing the direction and magnitude of the magnetic field measured at arm height on an 0.6 meter grid. Red points could not be measured due to the presence of obstacles and were interpolated from the surrounding measurements.

Localization of the user was performed by the commercial UbiSense system, described in section III-C, with antennas placed at the corners of the region and an additional two at the midpoints of the longer edges.

The user's wrist orientation was sensed by the inertial and magnetic sensors in the WristQue, as described in section III-D. The magnetic field in the space was measured where possible on an 0.6 meter grid at standing height, shown in fig. 5. In some places, due to the presence of furniture or other obstacles, it was necessary to vary the height at which the magnetic field was sampled. Locations at which it was impossible to sample are marked in red; these values were interpolated from the surrounding points in order to obtain a uniform grid. This grid was used to correct the heading of WristQue's orientation based on its current location in the space.

1) System accuracy test: To evaluate the performance of the pointing system, a user was instructed to stand at 144 different locations within the room and to point at a fixed target on the wall. At each location, the user's position and wrist orientation as reported by the system were sampled 20 times, and the intersection of the pointing ray with the wall plane was computed for each sample. The error distance from the center of the target was computed for each sample. The plots in fig. 6 show, for each location in the space where it was possible to stand and point, the percentage of samples where the user hit the target. Target sizes of 1,3, and 6 meters in diameter are shown.

This experiment is affected by both the error in the WristQue pointing system and the user's ability to point precisely at a target across the room. The results show that the pointing interface should work to select objects that are relatively large or relatively well-separated (more than a few meters) such as banks of light fixtures on the ceiling. For 


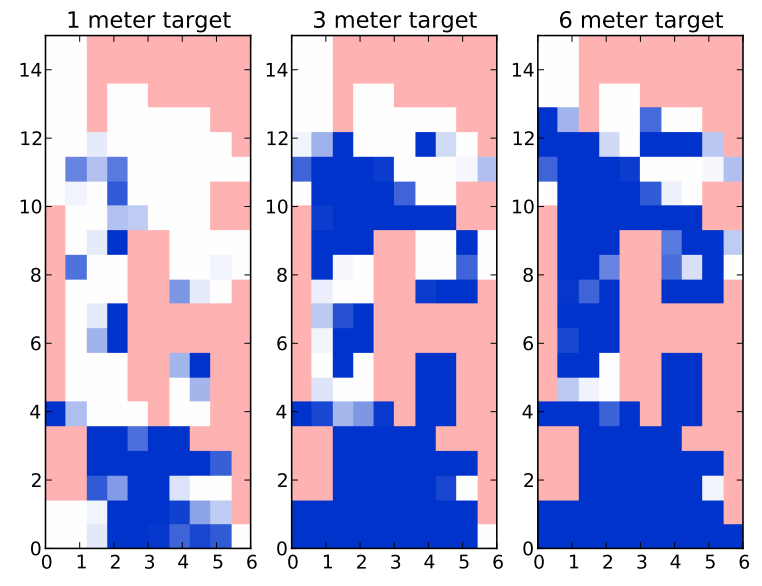

Fig. 6. Pointing system accuracy, shown on a map of the space (corresponding to the area shown with magnetic field data in fig. 5). Red areas contain furniture or equipment, preventing testing at that location. Blue shading indicates the percentage of pointing attempts from the location that hit targets that were 1,3 , and 6 meters in diameter. The target was located at $(3,0)$ in the plots. Units are in meters.

selecting smaller objects at a distance, providing feedback that indicates where the system estimates the user is pointing, so the user may make adjustments in the same way as a mouse cursor, will likely be important.

The lab space in which this test was conducted is a particularly challenging environment. The space is relatively dense with furniture, lab equipment, and other clutter, some of which had been moved since the magnetic field calibration data was taken.

2) Lighting control demo: The pointing system was extended to allow control over the lighting in the room. The lighting system in the space is a computer-controlled system found in many newer commercial buildings. Three tracks spaced approximately 3 meters apart run the length of the space, with fluorescent fixtures placed less than a meter apart into the track. Each half of each track can be independently controlled, creating six lighting zones completely within the area covered by the localization system (the area indicated in fig. 5). An additional two tracks, perpendicular to the rest, run through the space at the bottom of fig. 5. These are outside of the area covered by localization, but can be easily pointed at from it. The pointing demonstration allowed the user to dim these eight total lighting zones.

To take control, the user raises his arm. The low-power accelerometer detects this change in orientation and switches the WristQue into interaction mode, in which the full IMU is powered on. The user then performs a "start pointing" gesture by shaking his wrist, which the system recognizes to confirm that the user wishes to take control and is not just raising his arm. If this gesture is not detected within a few seconds, then the WristQue leaves interaction mode to conserve battery power. The gestures in this demo are recognized using the tools in [18]. The shake gesture is identified by taking raw accelerometer data, computing the derivative of each axis with a small deadzone, storing the results in a buffer. The number of zero-crossings in the buffer and the maximum magnitude are used as inputs to a K-nearest-neighbor classifier, which was trained on several seconds of shaking and normal, non-shaking movement.

Once the system has confirmed that the user wishes to take control, it waits for the user to point at one of the rectangular regions in the ceiling plane that corresponds to a bank of lights. When the user stops moving, the bank of lights at which the user is pointing is selected and briefly flashes to provide feedback to the user that he is now in control. If the user sees that the system has incorrectly identified his intended control target, or he changes his mind, he can once again shake his wrist to clear the selection and point at another target.

Once selected, the dimmer for the lights can then be controlled by a continuous gesture where the user rotates his wrist. The system considers the rotation of the wrist around the axis of the user's arm, such that rotating one's wrist and hand counterclockwise as far as easily possible will turn the lights off completely, and turning one's wrist completely clockwise will turn on the lights to full brightness. Finally, when the user is satisfied with the adjustment, he performs a final "stop pointing" gesture, lowering his arm and pointing toward the floor, to signal that he is done. This causes the system to release control of the lights and the WristQue leaves interaction mode to conserve power. The system will revert the control value to several samples before the stop gesture is detected so that wrist rotation during the stop gesture itself is not interpreted as control input.

Several subjects who had not previously used the pointing interface were able to control the lights after brief instruction.

\section{Adaptive lighting}

The pointing-based interface described above shows how the WristQue can be used to enable users to manually adjust the lighting to their preferences. However, lighting conditions change throughout the day as varying amounts of sunlight enters through windows and contributes to the overall lighting. Users also move around the space, and may wish for their lighting preferences to follow them. Furthermore, as lighting accounts for $22 \%$ of US energy usage [19] there is strong motivation to minimize environmental impact and cut operational costs by conserving energy in lighting. At the same time, building occupants should not be made to work under insufficient light.

WristQue builds on the work in [20], which describes a solid-state lighting system where each individual light source can be modulated at rates fast enough that there is no visible flicker. In [20], one light source to be measured is pulse-width modulated at a different fundamental frequency than the others, with the test source time-multiplexed between the available fixtures until a measurement has been obtained for each. In updating the experiment for the WristQue, the modulation was changed so that each source operates with a different PWM fundamental frequency, such that the harmonics do not overlap. This removes the need for the sensor to be time-synchronized with the controller.

The WristQue's monochrome light sensor periodically records a buffer of 2048 samples at $12 \mathrm{kHz}$ and transmits it back over the network. The server then performs an FFT on the buffer to separate out the contribution from each light source at the user's present location. With prior knowledge relating the fixtures' PWM duty cycle, current consumption, and illuminance, the system can solve a linear program to 
provide the desired light output at the user's location while minimizing power consumption.

\section{Personalized comfort control}

Nearly all HVAC control systems are based on manually adjusted and programmed thermostats that regulate temperature at arbitrary locations on the wall, rather than where people are actually using the space. [21] describes a system that moves the sensing into a wearable device that measures temperature and humidity at the wearer's location, and learns the wearer's preferences as the user indicates too hot, too cold, or neutral with the device's interface. WristQue performs the temperature, humidity, and location sensing required to implement this system.

\section{FUTURE WORK}

\section{A. Fusion with infrastructure-based sensors}

The most significant challenge in implementing a robust indoor pointing-based interface on the WristQue is determining the user's heading. The use of the earth's magnetic field, as discussed earlier, is not straightforward inside of buildings. Significant time was required for collecting the magnetic field calibration data used in the experiments described in this paper. Camera and depth sensors deployed in the environment could be combined with the WristQue's internal sensing and robust identification of the wearer to provide a better estimate of the position and orientation of the user's arm (and thus the WristQue) when the user is within view of the cameras.

\section{B. Additional control inputs}

The WristQue currently implements buttons and the pointing/gestural interfaces as input modalities for the user to take control. Additional input modalities are also possible. The WristQue hardware includes a controller for capacitive touch sensing, which has not yet been used. This could provide additional virtual buttons, as well as sliders that could be used for continuous controls. Replacing the physical buttons with touch-sensitive areas could allow the WristQue to be made smaller and better sealed, moving closer to the goal of being as wearable as possible.

Another possible input modality could use the accelerometer (and/or the addition of a microphone) to develop a control language based on tapping and scratching the device. This could be used, for example, to easily activate or cancel specific interaction modes.

\section{Magnetic field calibration}

The magnetic field calibration issue remains significant, with a large initial time investment required to produce the calibration data. Other approaches for determining indoor heading or at least minimizing the calibration time required are being investigated. Better interpolation methods could reduce the number of points that need to be sampled for calibration. Location and IMU data may also be used to automatically learn the magnetic field.

\section{CONCLUSION}

We have presented WristQue, a new wearable sensor platform that senses the user's location, wrist orientation, and environment. The experiments presented show how WristQue can both improve automatic control of infrastructure systems and provide a more intuitive and readily accessible interface to the myriad of controllable devices in the user's environment based on pointing for selection and gestures for control.

\section{REFERENCES}

[1] R. Jota, M. A. Nacenta, J. A. Jorge, S. Carpendale, and S. Greenberg, "A comparison of ray pointing techniques for very large displays," in Proceedings of Graphics Interface 2010, Ottawa, Ontario, Canada: Canadian Information Processing Society, 2010, pp. 269-276.

[2] C. Narayanaswami and M. Raghunath, "Application design for a smart watch with a high resolution display," in Wearable Computers, The Fourth International Symposium on, 2000, pp. 7 -14. DOI: 10.1109/ ISWC. 2000.888452

[3] J. McHugh, "Wrist-top revolution," Wired, vol. 11.04, Apr. 2003. [Online]. Available: http://www. wired.com/wired/archive/11.04/ fossil.html.

[4] U. Maurer, A. Rowe, A. Smailagic, and D. Siewiorek, "Ewatch: a wearable sensor and notification platform," in Wearable and Implantable Body Sensor Networks, 2006. BSN 2006. International Workshop on, 2006, 4 pp. -145 . DOI: 10.1109/BSN.2006.24.

[5] Ez430-chronos, [Online]. Available: http://processors.wiki.ti.com/ index.php/EZ430-Chronos (visited on 07/11/2012).

[6] B. Mayton, G. Dublon, S. Palacios, and J. A. Paradiso, "Truss: tracking risk with ubiquitous smart sensing," in IEEE Sensors, IEEE, 2012.

[7] T. Choudhury, S. Consolvo, B. Harrison, J. Hightower, A. LaMarca, L. LeGrand, A. Rahimi, A. Rea, G. Bordello, B. Hemingway, P. Klasnja, K. Koscher, J. Landay, J. Lester, D. Wyatt, and D. Haehnel, "The mobile sensing platform: an embedded activity recognition system," Pervasive Computing, IEEE, vol. 7, no. 2, pp. $32-41$, 2008, ISSN: 1536-1268. DOI: 10.1109/MPRV.2008.39.

[8] A. Pantelopoulos and N. Bourbakis, "A survey on wearable sensorbased systems for health monitoring and prognosis," Systems, Man, and Cybernetics, Part C: Applications and Reviews, IEEE Transactions on, vol. 40, no. 1, pp. $1-12$, 2010, ISSN: 1094-6977. DOI: 10.1109/ TSMCC.2009.2032660.

[9] A. Smailagic and D. Siewiorek, "Application design for wearable and context-aware computers," IEEE Pervasive Computing, vol. 1, pp. 2029, 2002.

[10] S. Greenberg, N. Marquardt, T. Ballendat, R. Diaz-Marino, and M. Wang, "Proxemic interactions: the new ubicomp?" interactions, vol 18, no. 1, pp. 42-50, Jan. 2011, ISSN: 1072-5520. DOI: 10.1145/ 1897239.1897250. [Online]. Available: http://doi.acm.org/10.1145/ 1897239.1897250

[11] B. D. Mayton, "Wristque: a personal sensor wristband for smart infrastructure and control," Master's thesis, Massachusetts Institute of Technology, 2013

[12] M. Quigley, K. Conley, B. P. Gerkey, J. Faust, T. Foote, J. Leibs, R Wheeler, and A. Y. Ng, "Ros: an open-source robot operating system," in ICRA Workshop on Open Source Software, 2009. [Online]. Available: http://www.willowgarage.com/sites/default/files/icraoss09-ROS.pdf.

[13] C. Nerney, "Qualcomm reveals details of peanut wireless technology," IT World, Sep. 22, 2010.

[14] F. Belloni, V. Ranki, A. Kainulainen, and A. Richter, "Angle-based indoor positioning system for open indoor environments," in Positioning, Navigation and Communication, 2009. WPNC 2009. 6th Workshop on, 2009, pp. 261 -265. DOI: 10.1109/WPNC.2009.4907836.

[15] Real-time location systems and geospatial consulting - ubisense, [Online]. Available: http://www.ubisense.net/ (visited on 09/03/2012).

[16] S. Madgwick, A. Harrison, and R. Vaidyanathan, "Estimation of imu and marg orientation using a gradient descent algorithm," in Rehabilitation Robotics (ICORR), 2011 IEEE International Conference on, 2011, pp. 1 -7.

[17] J. Chung, M. Donahoe, C. Schmandt, I.-J. Kim, P. Razavai, and M. Wiseman, "Indoor location sensing using geo-magnetism," in Proceedings of the 9th international conference on Mobile systems, applications, and services, Bethesda, MD, USA: ACM, 2011, pp. 141-154.

[18] N. Gillian. Gesture recognition toolkit, [Online]. Available: http://www. nickgillian.com/software/grt (visited on 02/01/2013).

[19] N. C. Inc., "Solid-state lighting research and development portfolio: multi-year program plan fy'09-fy' 15," Lighting Research, Development Building Technologies Program, Office of Energy Efficiency, and Renewable Energy, US Department of Energy, 2009.

[20] B. Lee, M. Aldrich, and J. Paradiso, "Methods for measuring work surface illuminance in adaptive solid state lighting networks," in Society of Photo-Optical Instrumentation Engineers (SPIE) Conference Series, vol. 8123, 2011, p. 22.

[21] M. Feldmeier and J. Paradiso, "Personalized hvac control system," in Internet of Things (IOT), 2010, 2010, pp. $1-8$. 